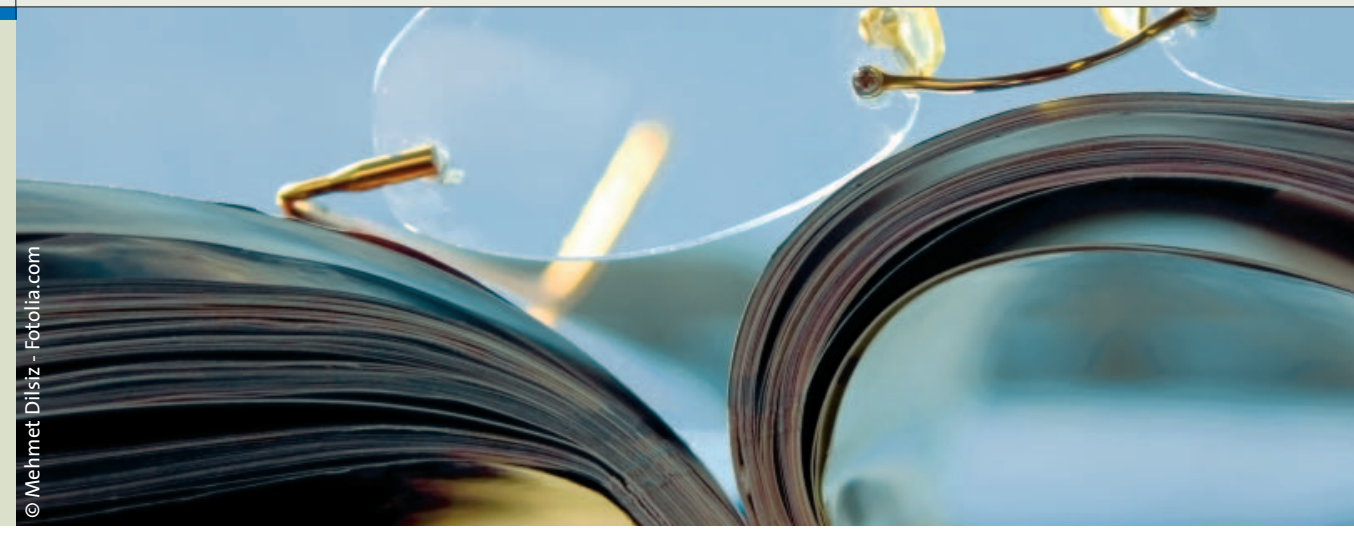

\section{Sensibilisiert - aber nicht allergisch}

Bei jungen Kindern sind Sensibilisierungen gegen Nahrungsmittelallergene häufig. Inwieweit dies zu einer klinischen Allergie führt und welche Rolle das atopische Ekzem dabei spielt, sollte eine dänische Studie klären.

$D$ ie Studienpopulation bestand aus 562 Kindern, die monatlich zwischen 1998 und 1999 rekrutiert wurden. Von Geburt an erfolgten in regelmäßigen Abständen Interviews mit den Eltern sowie körperliche Untersuchungen, Pricktests und IgE-Bestimmungen bei den Kindern. Innerhalb von sechs Jahren wurde bei 65 Kindern (12\%) von einer atopischen Dermatitis oder urtikariellen und rhinitischen Beschwerden in Zusammenhang mit Nahrungsmitteln berichtet. Bei 66 Kindern mit Verdacht auf Nahrungsmittelallergie erfolgten orale Provokationstests.

Bei 20 Studienteilnehmern konnten die Allergien klinisch bestätigt werden. Dies entsprach einer 6-Jahres-Indizidenz von 3,7\%. Im zeitlichen Verlauf unterschieden sich die Inzidenzen deutlich (sechs Monate: 3,6\%; ein Jahr: 1,3\%; 18 Monate: 3,6\%; drei Jahre: 3,4\%; sechs Jahre: $1,2 \%)$. Neu aufgetretene Fälle wurden ab einem Alter von drei Jahren nicht mehr beobachtet. Nach sechs Jahren bestand keine Allergie mehr gegen Milch- und zu 80\% nicht mehr gegen Hühnereiweiß. Die ersten Fälle von Erdnussallergie wurden erst ab 18 Monaten nachgewiesen. Verdachtsfälle auf Fleisch-, Fisch- oder Sojaallergie bestätigten sich nicht.

Insgesamt zeigten sich bei $47 \%$ der Studienteilnehmer Sensibilisierungen. 92\% dieser Kinder zeigten jedoch keinerlei Unverträglichkeitsreaktionen. 16 der 20 Kinder mit bestätigten Nahrungsmittelallergien waren zusätzlich sensibilisiert gegen weitere Allergene, die sie problemlos tolerierten. Unter 122 Kindern mit atopischer Dermatitis war in 18 Fällen eine Nahrungsmittelallergie nachweisbar. Kinder mit atopischer Dermatitis zeigten vergleichsweise weder häufiger Sensibilisierungen noch ein höheres Gesamt-IgE.

Fazit: Es besteht eine große Diskrepanz zwischen den Raten an Sensibilisierungen bzw. atopischer Dermatitis und klinisch bestätigten Nahrungsmittelallergien. Standardisierte Provokationstests sind erforderlich, um Nahrungsmittelallergien exakt zu diagnostizieren.

af

Eller E et al. Food allergy and food sensitization in early childhood: Results from the DARC cohort. Allergy 2009; 64: 1023-9.

\title{
Bei wem schlägt Stress auf die Haut durch?
}

\section{Stressfaktoren können Psoriasis-Schübe induzieren. Doch offenbar ist die Stress-Reaktivität individuell sehr unterschiedlich ausgeprägt. In einer Studie sind niederländische Forscher der Frage nachgegangen, bei wem genau Stress auf die Haut durch zu schlagen scheint.}

$\mathrm{V}$ iele Psoriatiker glauben, dass sich belastende Ereignisse und chronischer Stress ungünstig auf ihre Erkrankung auswirken. Rückblickend berichten etwa sechs von zehn Patienten über stressige Erlebnisse im Monaten vor einem akuten Psoriasisschub. Umgekehrt ist es allerdings schwierig, Schübe anhand des erlebten Alltagsstresses vorher zu sagen. Entsprechende Zusammenhänge erweisen sich meist als insgesamt eher schwach und von individuellen Unterschieden geprägt. Doch es scheint Patienten-Subpopulationen zu geben, bei denen sich stressige Zeiten stärker auf den Hautzustand auswirken als bei anderen.

In einer sechsmonatigen, prospektiven Studie an 62 Psoriatikern evaluierten Kollegen um E.W. Verhoeven, Nijmegen, mit Hilfe mehrerer Fragebögen belastende
Lebensereignisse, typischen Verhaltensund Denkgewohnheiten und korrelierten die Ergebnisse mit den PASI.

Fazit: Es zeigte sich, dass Stress vor allem für Menschen, die sich viele Sorgen wegen ihrer Krankheit machen und die sich viel kratzen, von Bedeutung ist. In Zeiten, in denen sie stark unter Stress stehen, verstärken sich diese Angewohnheiten und die Haut verschlechtert sich in den folgenden vier Wochen signifikant. Auch der Juckreiz wird dann noch schlimmer. isi

Verhoeven EW et al. Individual differences in the effect of daily stressors on psoriasis: a prospective study. Br J Dermatol 2009,161 (2): 295-9. 